\title{
L'inventaire de la voie ferrée Andelot - La Cluse ou une ligne en quête d'auteur
}

An inventory of the Andelot-La Cluse railway or a line in search of an a uthor

\section{Laurent Poupard}

\section{OpenEdition}

\section{Journals}

Édition électronique

URL : https://journals.openedition.org/rhcf/720

DOI : $10.4000 /$ rhcf.720

\section{Éditeur}

Rails \& histoire

\section{Édition imprimée}

Date de publication : 1 novembre 2009

Pagination : $53-74$

ISSN : 0996-9403

Référence électronique

Laurent Poupard, "L'inventaire de la voie ferrée Andelot - La Cluse ou une ligne en quête d'auteur », Revue d'histoire des chemins de fer [En ligne], 40 | 2009, mis en ligne le 01 novembre 2011, consulté le 22 avril 2022. URL : http://journals.openedition.org/rhcf/720 ; DOI : https://doi.org/10.4000/rhcf.720 
Laurent POUPARD

\section{L'inventaire de la voie ferrée Andelot - La Cluse ou une ligne en quête d'auteur}

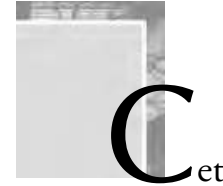

ette communication traite de l'étude d'une voie ferrée modeste reliant Andelot-en-Montagne dans le Jura à Montréal-La Cluse dans l'Ain, mais qui, desservant le Haut-Jura, présente une forte concentration d'ouvrages d'art, principalement dans la région de Morez - Saint-Claude. Elle présente la méthode retenue pour réaliser un inventaire de ce patrimoine ferroviaire (à l'origine d'une publication ${ }^{1}$ ), les problèmes rencontrés et, surtout, pose la question de l'identification des auteurs de cette ligne.

1- Laurent Poupard, La Ligne des Hirondelles. Un chemin de fer entre ciel et terre, d'Andelot à La Cluse (Jura-Ain), photogr. Yves Sancey, cartogr. André Céréza, Conseil régional de Franche-Comté, Direction de l'inventaire du patrimoine, Lyon, Lieux Dits, coll. " Parcours du Patrimoine », $n^{\circ} 336$, juillet 2008, 72 pages. 


\section{La ligne Andelot - La Cluse}

\section{Histoire}

Ouverte dans son entier en 1912, la voie ferrée Andelot - La Cluse relie les deux lignes existantes de :

- Dole - Neuchâtel via Mouchard, Andelot-en-Montagne et Pontarlier (ligne du «Franco-Suisse »), dans le Jura et le Doubs, inaugurée en 1862 ;

- Bourg-en-Bresse - Genève via La Cluse, Bellegarde et Culoz (ligne du « Haut-Bugey»), dans l'Ain, exécutée jusqu’à La Cluse en 1877 et jusqu’à Bellegarde en 1882 (fig. 1).

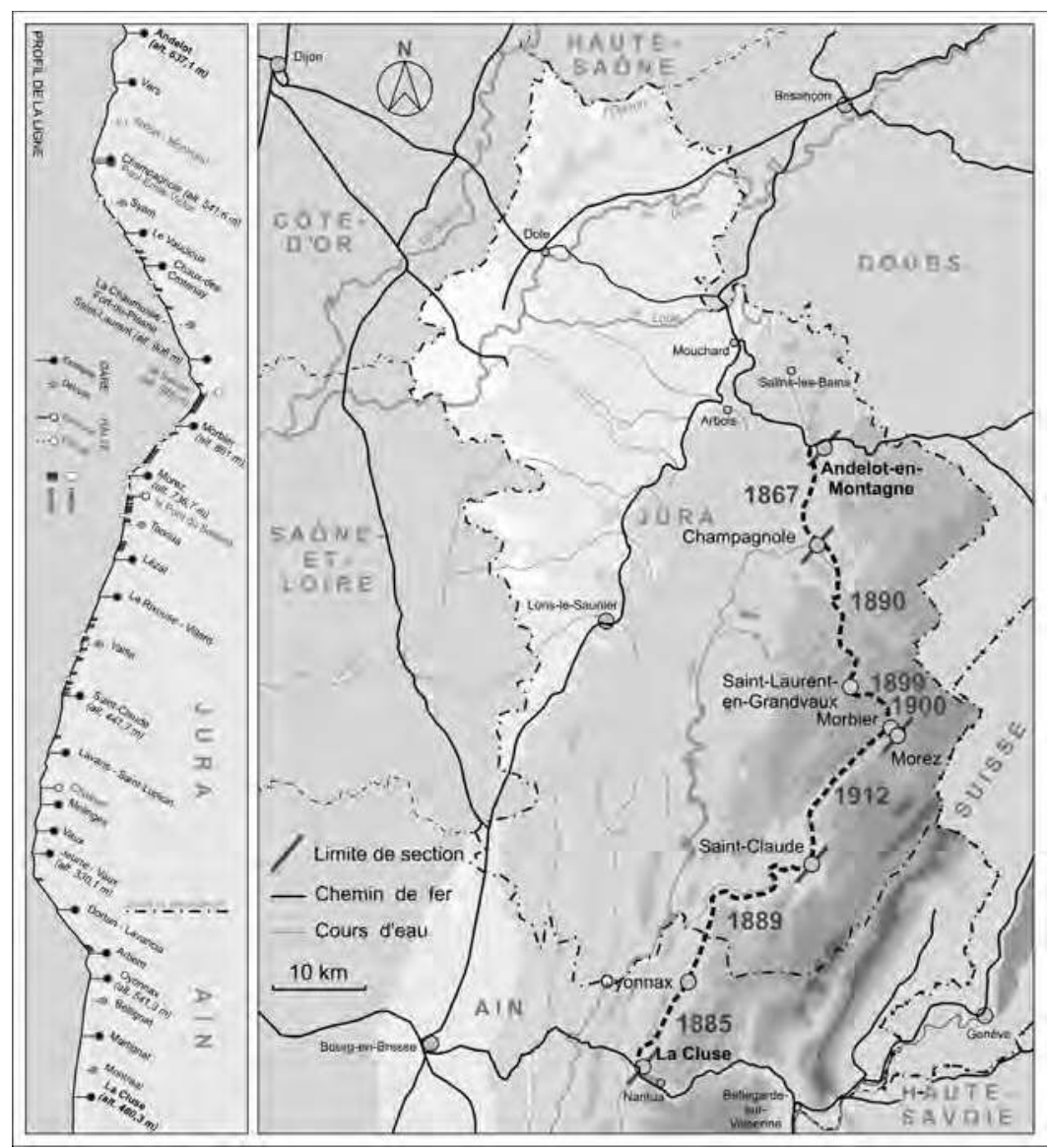

- Figure 1. Carte de localisation et profil de la ligne. Dessin André Céréza ${ }^{\odot}$ Région Franche-Comté, Inventaire du patrimoine, ADAGP, 2008 
Sa construction s'est poursuivie au gré des concessions faites au PLM :

- 1863 pour la section d'Andelot-en-Montagne à Champagnole (14 km), exécutée entre 1865 et 1867 ;

- 1883 pour la section de Saint-Claude à La Cluse $(42 \mathrm{~km})$, exécutée entre 1881 et $1889^{2}$;

- 1883 pour la section de Champagnole à Morez $(35 \mathrm{~km})$, exécutée entre 1881 et $1900^{3}$;

- 1901 pour la section de Morez à Saint-Claude $(24$ km), exécutée entre 1905 et $1912^{4}$.

Le retard dans la mise en ouvre de la partie méridionale de la ligne découle du refus renouvelé de l'État de prendre en charge une voie de montagne jugée peu rentable. La loi du 12 juillet 1865, donnant aux départements et aux communes l'opportunité d'établir eux-mêmes ou de concéder des voies ferrées, semble être la réponse. Le conseil général du Jura ne souhaite cependant pas l'adopter : en effet, le financement qui en découle obligerait à réaliser cette voie - classée d'intérêt local - avec un écartement réduit alors que la première section est à écartement normal, d'où des ruptures de charges jugées inacceptables. Alors que de guerre lasse, le temps passant, il se résigne à cette solution, la loi Freycinet du 17 juillet 1879 vient combler ses attentes : portant la création d'un " troisième réseau " (à voie normale) destiné à desservir l'ensemble des sous-préfectures et un maximum de chefs-lieux de cantons, elle retient sous le $\mathrm{n}^{\circ} 119$ la réalisation d'une ligne de Champagnole à ou près Ambérieu, par La Cluse, avec embranchement sur Morez et embranchement de Verges à Jeurre. En 1880, deux lois déclarent d'utilité publique les sections de Champagnole à Morez et de Saint-Claude à La Cluse, et autorisent l'État à entreprendre leur construction sans plus attendre.

Si l'exécution de la deuxième section ne pose pas de problèmes majeurs, il n'en est pas de même de celle courant de Champagnole à Morez, qui exige de plus grands délais. Ainsi, longtemps considéré comme rédhibitoire, le nombre d'ouvrages d'art rendus nécessaires par la topographie du tronçon de Morbier à Morez porte le coût de ces cinq kilomètres de voie à près d'un million de francs or au kilomètre, le plus élevé de France à cette époque (fig. 2).

2- Travaux scindés en deux tronçons : La Cluse - Oyonnax (12 km) et Saint-Claude - Oyonnax (30 km), ouverts respectivement en 1885 et 1889.

3- Travaux scindés en trois tronçons : Champagnole - Saint-Laurent-en-Grandvaux (22 km) de 1881 à 1890, Saint-Laurent-en-Grandvaux - Morbier (8 km) de 1892 à 1899, Morbier - Morez (5 km) de 1894 à 1900.

4- Ligne concédée au PLM à titre éventuel dès 1886 sous réserve de la déclaration d’utilité publique à venir, chose faite en 1901 seulement. 


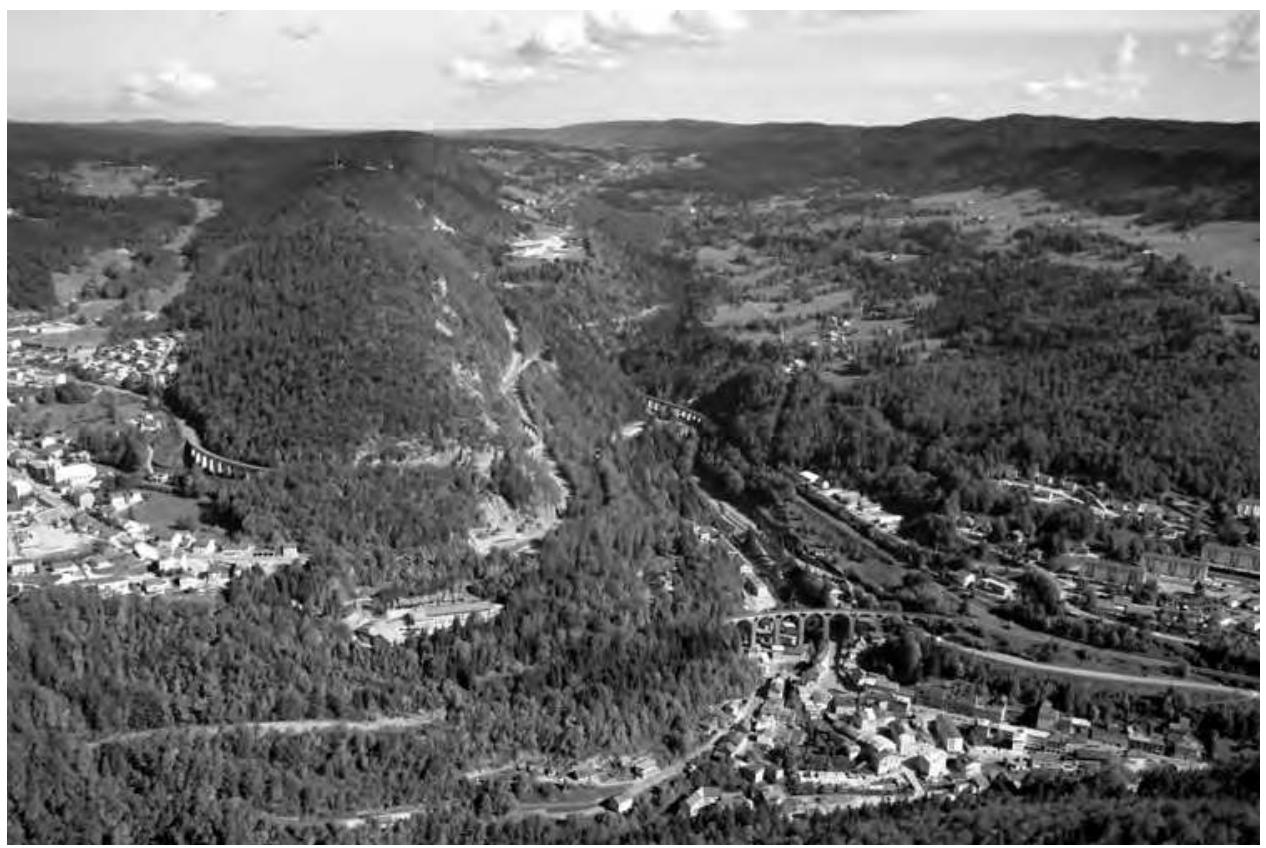

- Figure 2. Les ouvrages d'art de la zone Morbier - Morez. De Morbier à gauche à Morez à droite, la voie enchaîne les ouvrages, dont les principaux sont : viaduc de Morbier, tunnel des Crottes, viaducs des Crottes puis de Romand, tunnels des Frasses et du Pâturage, viaducs de l'Evalude et de la Source. Le viaduc de Morez (Séjourné, 1912), au premier plan, se trouve sur la section Morez - Saint-Claude. Cliché Jean-Pierre Bévalot ๑ Région Franche-Comté, Inventaire du patrimoine, ADAGP, 2005.

La fixation, au début des années 1890, du tracé de ce tronçon permet de relancer les études de la quatrième et dernière section, menant de Morez à Saint-Claude. Le milieu, tout aussi défavorable, oblige également à multiplier les ouvrages. Cependant, la réticence du conseil général à garantir une subvention de 40000 francs par kilomètre promise en $1876^{5}$ conduit le ministre des Travaux publics à en repousser l'exécution, jusqu'à l'obtention de l'accord souhaité en 1900. Raison pour laquelle la section en question n'est finalement déclarée d'utilité publique et concédée au PLM qu'en 1901 (au titre d'une modalité de l'art. 1 des conventions de 1883, qui fait obligation au PLM d'accepter les concessions de $600 \mathrm{~km}$ de lignes non dénommées à désigner d'un commun accord avec l'État).

L'ouverture à l'exploitation de cette section, le 10 août 1912, marque l'achèvement de la ligne Andelot - La Cluse, quarante-sept ans après le premier

5- Du fait, objecte-t-il, d'un «trop long ajournement de l'exécution de cette ligne », alors qu'il l'avait pourtant consentie pour les deux sections de Champagnole - Morez et de Saint-Claude - La Cluse, déclarées d'utilité publique en 1880. 
coup de pioche ! Cette durée témoigne bien du peu d'empressement du concessionnaire (le PLM) et de l'État à réaliser une ligne dont la rentabilité est dès l'origine jugée douteuse.

\section{La voie et son patrimoine en chiffres}

C'est la voie ferrée franc-comtoise qui comporte le plus grand nombre d'ouvrages d'art : près de 700 pour la seule partie jurassienne selon la SNCF, soit en moyenne un tous les $140 \mathrm{~m}$ ! C'est aussi une ligne qui, quoique relativement bien préservée, a connu une certaine érosion de son patrimoine : destruction de gares et maisons de garde-barrière, fermeture de passages à niveau, etc. (tableau 1).

Tableau 1. Le patrimoine immobilier subsistant sur la ligne Andelot - La Cluse

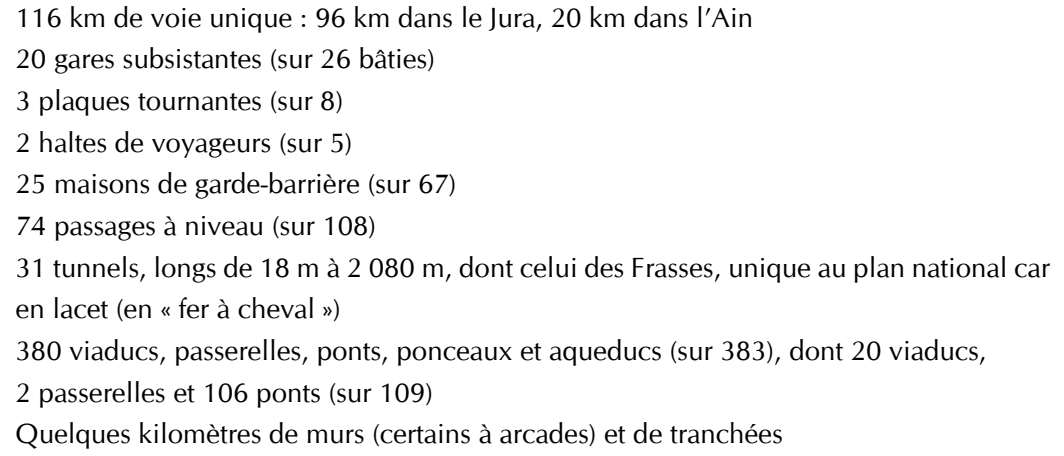

À ces ouvrages s'ajoutent aussi des œuvres que l'Inventaire répertorie comme objets mobiliers :

- objets concernant la signalisation : poteaux kilométriques et hectométriques, panneaux (lumineux ou non) à l'attention du mécanicien (conducteur du train) ou destinés à signaler les passages à niveau, etc. ;

- objets concernant la circulation des trains en gare : appareils de voie (improprement appelés " aiguillages ») et leurs systèmes de commande, appareils assurant la sécurité des manœuvres (serrure centrale, transmetteur de clefs, commutateur, tableau de contrôle optique), etc. ;

- objets concernant la gestion des voyageurs et des marchandises : mobilier de vente de billets et de salle d'attente, chariot à bagages, horloge, grue et portique roulant, balance à pont-bascule, etc. ;

- objets concernant la voie et son entretien : traverse et rail, machines à poser la voie, bourreuse à ballast, tirefonneuse, etc. ;

- objets concernant le matériel roulant et son service : locomotive, voiture, wagon, autorail, grue hydraulique, etc. 


\section{La démarche d'inventaire}

À l'origine, l'étude de la ligne a été envisagée comme un simple enregistrement des données : réalisation de notices et dossiers sur les seuls ouvrages d'art, gares et bâtiments remarquables ou typiques. Finalement, le travail réalisé vise à rendre compte de cette ligne comme d'un objet technique vivant, cohérent et fonctionnel. Cela a impliqué d'aller au-delà d'une simple analyse historique et typologique pour s'intéresser, par exemple, aux contraintes topographiques et techniques qui ont motivé la création de tel ou tel ouvrage, à l'évolution des instruments assurant la gestion et la sécurité des circulations, au parc du matériel roulant lui-même.

\section{La documentation}

L'Association pour l'histoire des chemins de fer en France (AHICF) constitue une bonne porte d'entrée pour aborder la documentation générale. Cette documentation est assez souvent abondante et disparate. Abondante car le monde ferroviaire a ses passionnés et les livres et articles de revue consacrés à telle ou telle ligne ne manquent pas. Pour Andelot - La Cluse, citons, par exemple, les ouvrages de Joseph Bécu ${ }^{6}$ et de Jean Cuynet ${ }^{7}$. Et n'oublions pas Internet qui offre de nombreuses monographies de lignes ${ }^{8}$. Disparate car la qualité en est très variable et peu atteignent le niveau de l'Histoire des chemins de fer de François Caron ${ }^{9}$ ou, dans une autre catégorie, du Larousse des trains et des chemins de fer de Clive Lamming ${ }^{10}$.

Les choses se compliquent lorsqu'il s'agit de retourner aux sources mêmes. Jusqu'à l'avènement de la SNCF en 1938, les grandes compagnies étaient indépendantes. D'où une multiplication des archives et de leur mode de gestion. Tout n'a pas été récupéré par la SNCF, loin s'en faut, et son centre d'archives du Mans ne conserve, par exemple, que 50 mètres linéaires de documents pour le PLM (du secteur Exploitation qui plus est) alors qu'il dispose de près de 1200 mètres linéaires pour la seule Compagnie de l'Est (et la région SNCF Est). Par ailleurs, la SNCF étant une administration an-

6- Joseph Bécu, Les Voies ferrées du Haut-Jura et des Carpates, [s.1.], 2000, 460 pages.

7- Jean Cuynet, Histoire du rail en Franche-Comté, Saint-Laurent-du-Var, La Régordane, 1989, 208 pages.

8- Site d'Elie Mandrillon (http://demophile.free.fr), comportant un important volet sur les voies métriques en Franche-Comté, notamment le réseau de la Compagnie générale des chemins de fer vicinaux.

9- François Caron, Histoire des chemins de fer en France, tome premier, 1740-1883, Paris, Fayard, 1997, 700 pages ; tome second, 1883-1937, 2005, 1029 pages.

10- Clive Lamming (dir.), Larousse des trains et des chemins de fer, Paris, Larousse, 2005, 509 pages. 
cienne et fortement hiérarchisée, les archives « manquantes " peuvent se trouver conservées en une multitude d'endroits, réparties entre établissements, directions, sous-directions ou bureaux quelconques, aux différents niveaux chargés de l'entretien des voies et ouvrages d'art à telle ou telle époque, etc. Et ceci est d'autant plus vrai que la ligne est encore en activité.

Les services d'archives départementales conservent généralement un nombre non négligeable de dossiers. En effet, le contrôle de l'État en matière de chemins de fer s'est très vite organisé, générant une procédure extrêmement précise à suivre pour obtenir une concession puis pour construire la voie ferrée correspondante. L'impulsion donnée en 1879 par le plan Freycinet a motivé la diffusion de circulaires rappelant cette procédure, qui se traduit par la constitution de tout un ensemble de dossiers normalisés :

- avant-projet (instruction du 14 janvier 1850);

- enquête d'utilité publique (ordonnance royale du 18 février 1834);

- projet de tracé et terrassements ;

- désignation des territoires traversés (loi du 3 mai 1841);

- enquêtes sur le nombre et l'emplacement des gares et stations (circulaires des 25 janvier 1854 et 9 août 1859);

- enquêtes parcellaires (loi du 3 mai 1841);

- projet d'exécution;

- arrêté de cessibilité, expropriation (loi du 3 mai 1841);

- prise de possession d'urgence des terrains non bâtis (loi du 3 mai 1841);

- estimations, cessions amiables ;

- offres légales, constitution et opérations du jury ;

- formalités relatives au paiement des indemnités;

- occupations temporaires de terrains (décret du 8 février 1868);

- récolement, réception et remise des ouvrages aux services intéressés (circulaire du 21 février 1877).

Cette procédure a conduit à la constitution des dossiers, la plupart du temps déposés par le service des Ponts et Chaussées, qui alimentent la soussérie $5 \mathrm{~S}$ Chemins de fer des archives départementales. Pour la ligne Andelot - La Cluse, ces dossiers, riches en documents généraux (sur la détermination du tracé, le type des gares, etc.), ne contiennent malheureusement pas de plan d'ouvrage individuel. En revanche, il convient de prêter attention aux décisions ministérielles qui valident (suivant l'avis du Conseil supérieur des Ponts et Chaussées) chacun des choix opérés au niveau de la ligne, tant pour sa construction initiale que par la suite (classement des passages à niveau, etc.). 
Souvent imprimés, les procès-verbaux des délibérations du conseil général (assemblée créée en 1789) sont, en tous cas, une source incontournable : cette assemblée n'a en effet cessé au XIX ${ }^{e}$ siècle de voir croître son rôle en matière d'aménagement ferroviaire (en liaison directe avec son devoir de financement). L'ingénieur en chef des Ponts et Chaussées responsable du service dans le département était en outre tenu de lui rendre compte des travaux réalisés, d'où une source fiable donnant deux fois par an un état d'avancement des constructions, puis de l'exploitation des voies ferrées.

\section{Un partenaire incontournable - la SNCF - et un document indispen- sable : le graphique de ligne}

L'étude a été menée avec la SNCF, partenaire incontournable même si elle n'est plus propriétaire des ouvrages. En effet, depuis 1997, ceux-ci relèvent de Réseau Ferré de France, établissement public industriel et commercial (EPIC).

Toutefois, dans la mesure où RFF confie l'entretien de son domaine à la SNCF et à ses agents, ces derniers sont les interlocuteurs naturels du chercheur pour l'étude de la ligne qui, si elle ne pose pas de problème depuis le domaine public, est plus encadrée dès lors que l'on pénètre dans l'emprise ferroviaire : les règles de sécurité de la SNCF exigent en effet que le chercheur soit accompagné par un agent habilité - d'où, en ce qui nous concerne, une campagne photographique sur les ouvrages d'art qui fut concentrée dans le temps.

La ligne étant encore en activité, il a été possible de se procurer, auprès des services chargés de son entretien, la copie d'un document indispensable intitulé Graphique, Graphique de ligne ou Carnet de ligne, parfois appelé aussi Carnet de poche (certainement du fait de ses dimensions : une quarantaine de centimètres de haut pour plusieurs mètres de longueur !) (fig. 3). Ce document est le seul moyen fiable et pratique de localiser les ouvrages existants. Il donne autour d'un schéma linéaire de la ligne, scandé par les points kilométriques (PK), des informations sur :

- les départements, arrondissements, cantons et communes traversées ;

- le profil (avec les déclivités) et les rayons de courbures;

- la nature du ballast et de la voie ;

- l'identification des bâtiments, abris, ouvrages d'art, passages à niveau, etc. 
C'est le seul document qui donne de la ligne - et a priori - une vision exhaustive (car tous les ouvrages y sont reportés) et géoréférencée (avec les PK). Cette dernière caractéristique est d'autant plus intéressante que ces ouvrages ne sont pas forcément reportés sur les fonds cartographiques habituellement utilisés : carte IGN au 1/25000 et plan cadastral. Faute de GPS, le système de repérage géographique propre à la voie, matérialisé par des bornes hectométriques et des poteaux kilométriques, est donc fondamental, d'autant plus qu'il constitue alors un référentiel partagé avec les agents de la SNCF.

Ce document permet en outre de réfléchir dès l'origine, si le service d'inventaire en est doté, à une exploitation de ces données à l'aide d'un système d'information géographique (SIG), outil on ne peut plus adapté au traitement de ce type de réseau tant dans une problématique de repérage / sélection (en amont de la rédaction des dossiers) que pour une restitution (en aval). Cette dernière pose d'ailleurs le problème très concret d'une cartographie donnant à voir $116 \mathrm{~km}$ de ligne sur un support papier.

\section{La question de la sélection}

La démarche habituelle de l'Inventaire veut que, pour une famille d'œuvres, tout soit vu et intégré dans une grille de repérage permettant la réalisation de typologies. Il devient alors possible d'opérer une sélection au sein de chaque type et, finalement, de restreindre l'étude à un certain pourcentage du corpus (idéalement compris entre 5 et $20 \%$ ). Cette démarche n'a pas pu être appliquée pour tout un ensemble de raisons :

- tout d'abord, les conditions de réalisation de l'étude pour les ouvrages d'art, concentrée sur les quelques jours de la campagne photographique et sans retour possible, ont constitué un obstacle majeur ;

- le nombre des ouvres, connu dans un second temps seulement, a joué : par exemple, il n’a pas été possible de voir les 252 aqueducs (ce qui aurait présenté un intérêt tout relatif) mais la sélection finale en a retenu 8 , à titre d'échantillonnage ;

- le fait que le corpus soit constitué d'une architecture sérielle et / ou fortement typée a été pris en compte ;

- de même, l'intérêt de chaque ouvrage a été jugé suivant un référentiel variable ; ainsi des 12 gares de $3^{\text {e }}$ classe ou assimilées, 10 subsistent : en étudier une ou deux aurait pu suffire pour représenter ce type à l'échelle de la ligne mais en revenant à un découpage purement géographique, au niveau du canton et - plus encore - de la commune, chacune devient unique et constitue un élément patrimonial marquant ; 


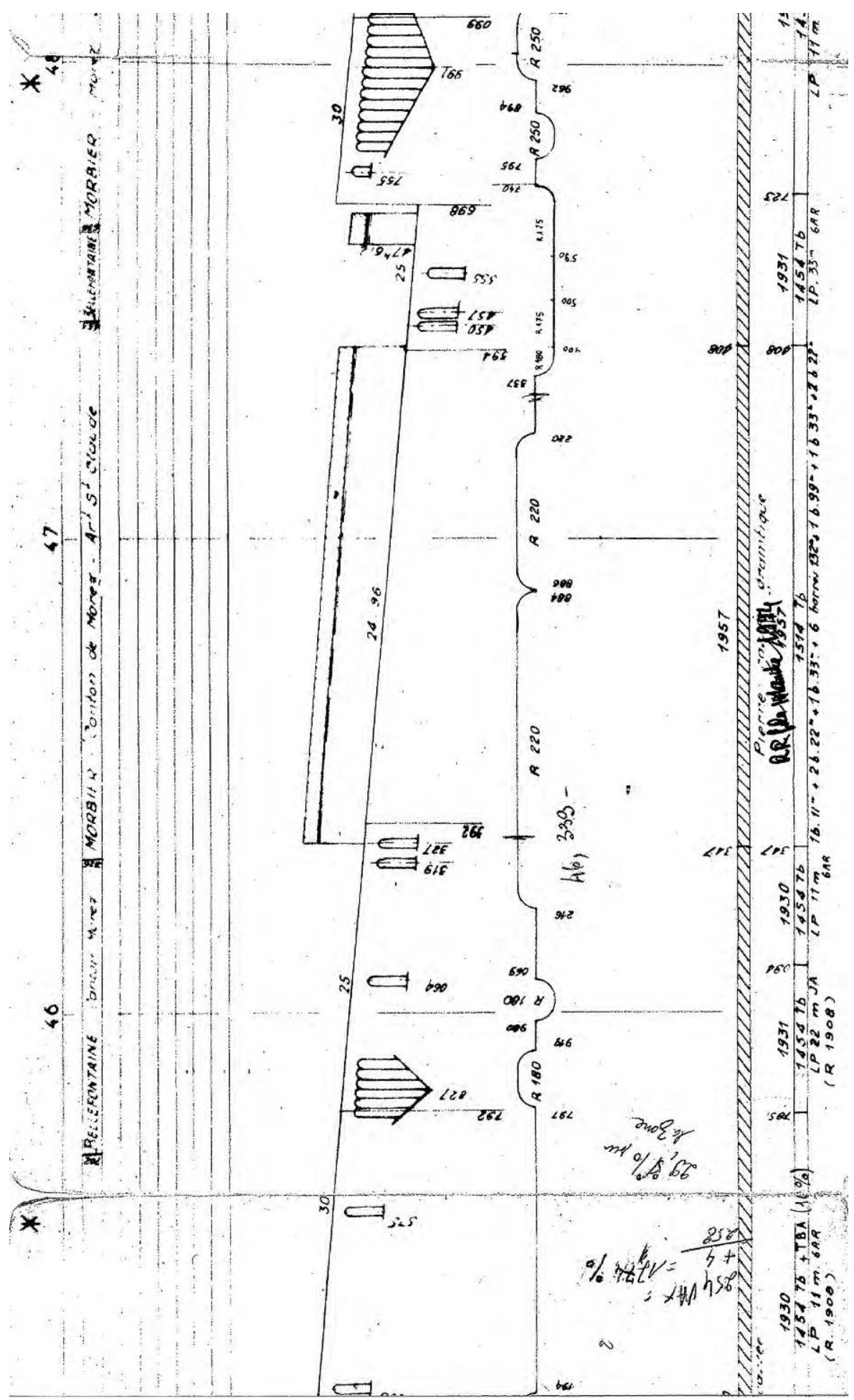


Laurent POUPARD

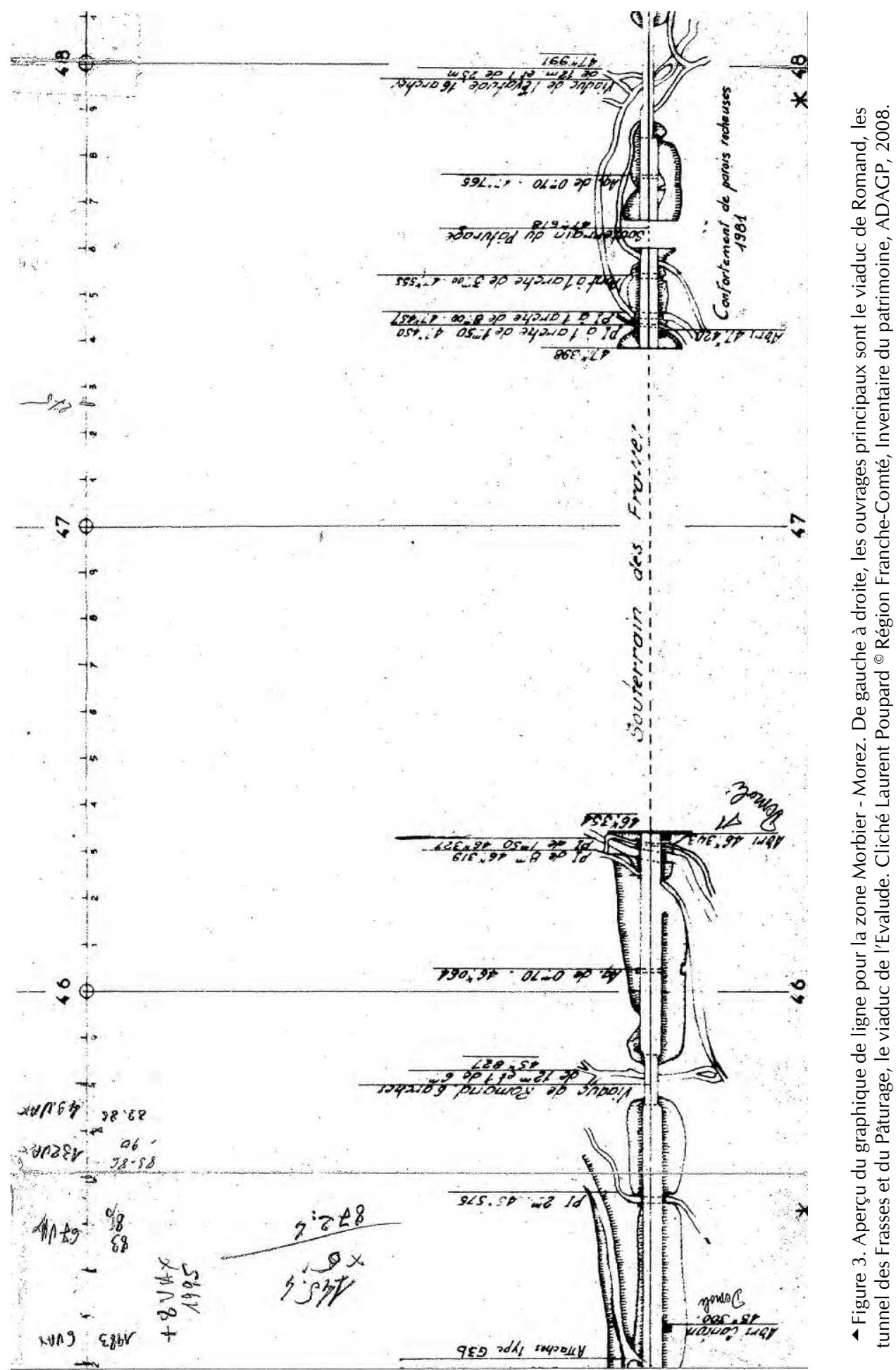

RHCF $40-63$ 
- le manque de critère scientifique pour certaines œuvres : sur quelle base sélectionner tel tunnel plutôt que tel autre ? Faut-il prendre le plus long ? Le plus court? Le plus courbe ? Le plus rectiligne ? Le plus en pente ? Etc.

Le résultat final est donc disparate du point de vue de la démarche repérage / sélection. Ainsi, les 20 viaducs ont tous été sélectionnés (car considérés comme autant d'œuvres uniques) alors que n'ont été retenus que 27 tunnels sur les 31 existants : ceux photographiés. 30 des 98 gares, haltes et maisons de garde-barrière édifiées ont été sélectionnées, sachant que les 20 gares subsistantes ont systématiquement été étudiées.

Cette sélection toute relative s'assume aisément si l'on envisage l'étude dans la perspective d'un recensement illustré, c'est-à-dire d'un enregistrement de l'intégralité du corpus. Par ailleurs, le nombre des dossiers (127) reste maîtrisé dans la mesure où certains d'entre eux traitent de plusieurs œuvres proches géographiquement et fonctionnellement liées : ainsi sur la commune du Vaudioux, au lieu-dit Malproche, le viaduc, le pont métallique et le tunnel qui se succèdent dans cet ordre sur moins de $400 \mathrm{~m}$ sont réunis en une seule notice.

\section{Modèles, plans type et auteurs}

S'il est un domaine où la pratique d'une architecture basée sur la répétition de plans type et de modèles a prévalu, c'est bien le domaine ferroviaire ce qui, du coup, pose des problèmes quant à l'identification des auteurs de la ligne et de ses ouvrages.

\section{Modèles d'ouvrages d'art}

Le ministre des Travaux publics Freycinet accompagne son programme par deux circulaires, en date des 28 juin 1879 et 26 avril 1880, qui permettent de « choisir, pour la rédaction des projets de construction de toute nature, des types parmi ceux qui présentent à la fois les conditions d'une bonne construction et le caractère d'une sage économie ${ }^{11}$. Il donne donc, sélectionnés parmi

\footnotetext{
11- Archives départementales du Jura (désormais : AD Jura) :

- Sp 2184 Ouvrages d'art. Recueil de modèles types, 1879-1880 [Recueil de types (plans, profils, ouvrages d'art) et de tableaux et procédés graphiques pour l'étude et la construction des chemins de fer. Annexé à la Circulaire ministérielle du 30 juillet 1879, par le Ministère des Travaux publics. Direction générale des Chemins de fer, 1879 [et] Recueil de types (maisons de gardes, stations : dispositions générales et bâtiments) pour l'étude et la construction des chemins de fer. Annexé à la Circulaire ministérielle du 26 avril 1880, par le Ministère des Travaux publics. Direction générale des Chemins de fer, 1880. Contient 5 types de maisons de gardes, 14 plans généraux de stations, 21 types de bâtiments de voyageurs, 5 types de bâtiments de marchandises] ;
} 
les modèles des différentes compagnies, des dessins d'aqueducs, de ponts, de maisons de gardes, de bâtiments voyageurs et d'entrepôts de marchandises, etc.

Le gabarit des tunnels est fixé : section ovoïde (résistant le mieux à la poussée des terres), largeur variant de 4,50 m (au niveau des rails) à $5,10 \mathrm{~m}$ au maximum, $6 \mathrm{~m}$ de hauteur au-dessus des rails et une cinquantaine de centimètres en dessous, voûte (légèrement surhaussée) en berceau plein-cintre.

Les caractéristiques des ponts ferroviaires sont précisées suivant la nature de l'obstacle franchi : ouverture minimale (largeur entre culées) de $8 \mathrm{~m}$ audessus d'une route nationale, $7 \mathrm{~m}$ pour une route départementale, $5 \mathrm{~m}$ pour un chemin vicinal de grande communication et $4 \mathrm{~m}$ pour un simple chemin vicinal. La hauteur libre est d'au moins $5 \mathrm{~m}$ sous clef de voûte et $4,30 \mathrm{~m}$ sous poutre, la largeur entre parapets de 4,50 m minimum pour une voie unique.

En matière de viaduc, les ingénieurs sont laissés libres car, pour des ouvrages de cette dimension devant résoudre un problème de franchissement à chaque fois différent, il ne peut y avoir de modèle absolu. Toutefois, ils sont quasiment tous réalisés suivant le même modèle ; seuls changent leur hauteur, leur longueur, le nombre et la largeur de leurs travées.

Les aqueducs ne sont pas oubliés : $60 \mathrm{~cm}$ d'ouverture pour $70 \mathrm{~cm}$ de hauteur au minimum. La distinction entre pont et aqueduc n'est d'ailleurs pas établie sur la fonction de l'ouvrage, mais sur ses dimensions. La SNCF parle d'aqueduc pour les passages inférieurs (sous la voie) jusqu'à $2 \mathrm{~m}$ de large, de ponceaux pour les ouvrages de 2 à $4 \mathrm{~m}$ et de pont (ou de viaduc) au-delà. Remise en question dans un premier temps, cette typologie a finalement été retenue lors de l'étude et systématisée, entraînant un changement de dénomination de certains des ouvrages qui d'aqueducs SNCF sont devenus des ponts pour l'Inventaire, et inversement.

\section{Plans type de bâtiments}

Les dispositions des gares et des bâtiments sont, elles aussi, évoquées par des plans type accompagnant les circulaires de Freycinet. Le PLM y est à l'honneur : à la différence des autres compagnies, chaque catégorie de bâtiment est illustrée par l'un de ses plans.

(suite de la note 11)

- Sp 2186 Recueils de formules et de types pour la construction des chemins de fer, 1879-1880 [Recueil de formules pour l'étude et la construction des chemins de fer. Annexé à la Circulaire ministérielle du 28 juin 1879, par le Ministère des Travaux publics. Direction générale des Chemins de fer, 1879 [et] Recueil de types (maisons de gardes, stations : dispositions générales et bâtiments) pour l'étude et la construction des chemins de fer. Annexé à la Circulaire ministérielle du 26 avril 1880, par le Ministère des Travaux publics. Direction générale des Chemins de fer, 1880]. 
La typologie des bâtiments de voyageurs de cette compagnie est exposée en 1881 par l'ingénieur Daveluy ${ }^{12}$ qui répartit ces constructions, à sous-sol et étage carré, en quatre classes en fonction du trafic :

$-1^{\text {re }}$ classe : plus de 150 voyageurs par jour et un public potentiel de plus de 12000 personnes; $308 \mathrm{~m}^{2}$ hors œuvre, bâtiment principal de 5 travées (avec un corps central de 3 travées) encadré par deux ailes en rez-de-chaussée (de 2 travées chacune), toits à croupes;

- $2^{\mathrm{e}}$ classe : de 80 à 150 voyageurs par jour et de 9000 à 12000 âmes ; $168 \mathrm{~m}^{2}, 5$ travées (avec un corps central de 3 travées), toit à croupes ;

- $3^{\text {e }}$ classe : de 30 à 80 voyageurs par jour et de 6000 à 9000 personnes ; $96 \mathrm{~m}^{2}, 3$ travées, toit à longs pans (fig. 4 et 5);

- $4^{\mathrm{e}}$ classe : moins de 30 voyageurs par jour et moins de 6000 habitants; $72 \mathrm{~m}^{2}, 2$ travées, toit à longs pans.

Plus répandue encore que la gare, la maison de garde-barrière modèle PLM présente une travée en façade et est coiffée d'un toit à longs pans. D'une superficie d'une quarantaine de mètres carrés $(8,07 \mathrm{~m} \times 5,10 \mathrm{~m})$, elle a une cave et quatre pièces de $12 \mathrm{~m}^{2}$ environ : cuisine et chambre au rez-de-chaussée, deux pièces à l'étage (fig. 6 et 7). Le prototype de ces habitations peut être trouvé dans les maisons d'éclusier : les canaux constituent le premier réseau qui, dès la fin du XVIII ${ }^{e}$ siècle, connut des essais, ensuite généralisés, de normalisation.

En 1855-1856, dans son Traité élémentaire des chemins de fer, l'ingénieur Auguste Perdonnet signalait déjà cette parenté, ainsi que les recherches en cours sur la taille à adopter :

"Les maisons de gardes, établies dans l'origine sur les chemins de fer en exploitation, ont été exécutées sur des dimensions et dans des conditions qui ont nécessité des dépenses assez considérables. Sur les chemins de fer du Nord, de Lyon et de Strasbourg, les maisons de gardes construites par l'État sont composées d'un rez-de-chaussée, d'un premier étage et d'une cave assez vaste ; elles sont revenues à 4000 francs en moyenne. Il en est de même des maisons d'éclusiers construites sur les divers canaux exécutés par le gouvernement.

Les Compagnies ayant reconnu qu'il y avait plus de sûreté et en définitive plus d'économie à établir des maisons de gardes à tous les passages à niveau pour que le service puisse en être fait par les femmes des poseurs employés à l'entretien, et le nombre des maisons de gardes étant appelé à devenir plus considérable que dans l'origine, ont recherché si l'on ne pourrait pas établir des bâtiments d'une importance moindre et en conséquence d'une dépense beaucoup plus restreinte ${ }^{13}$."

12- Daveluy, « Dimensions à donner aux bâtiments de voyageurs dans les gares », Revue générale des chemins de fer, février 1881, p. 116-119: 1 pl. h.t.

13- Auguste Perdonnet, Traité élémentaire des chemins de fer, Paris, Langlois et Leclercq, 1855-1856, vol. 2, p. 110. 


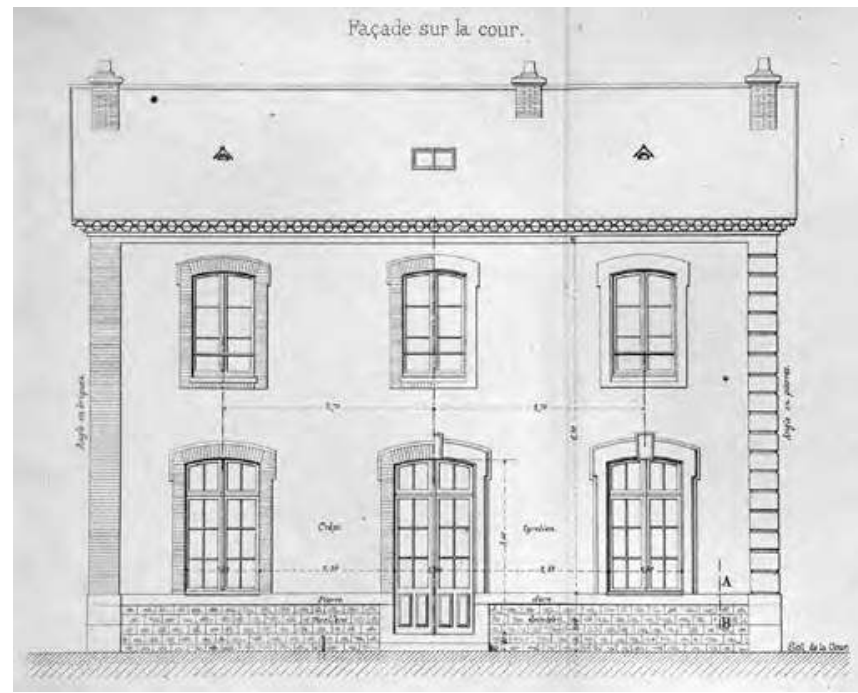

- Figure 4. Bâtiment des voyageurs pour Station de $3^{\mathrm{e}}$ classe. Type de la Cie de Paris-Lyon-Méditerranée. Dessin imprimé, s.n., s.d. [1880], échelle 1:50. AD Jura : Sp 2184. Cliché Yves Sancey ${ }^{\circledR}$ Région FrancheComté, Inventaire du patrimoine, ADAGP, 2008

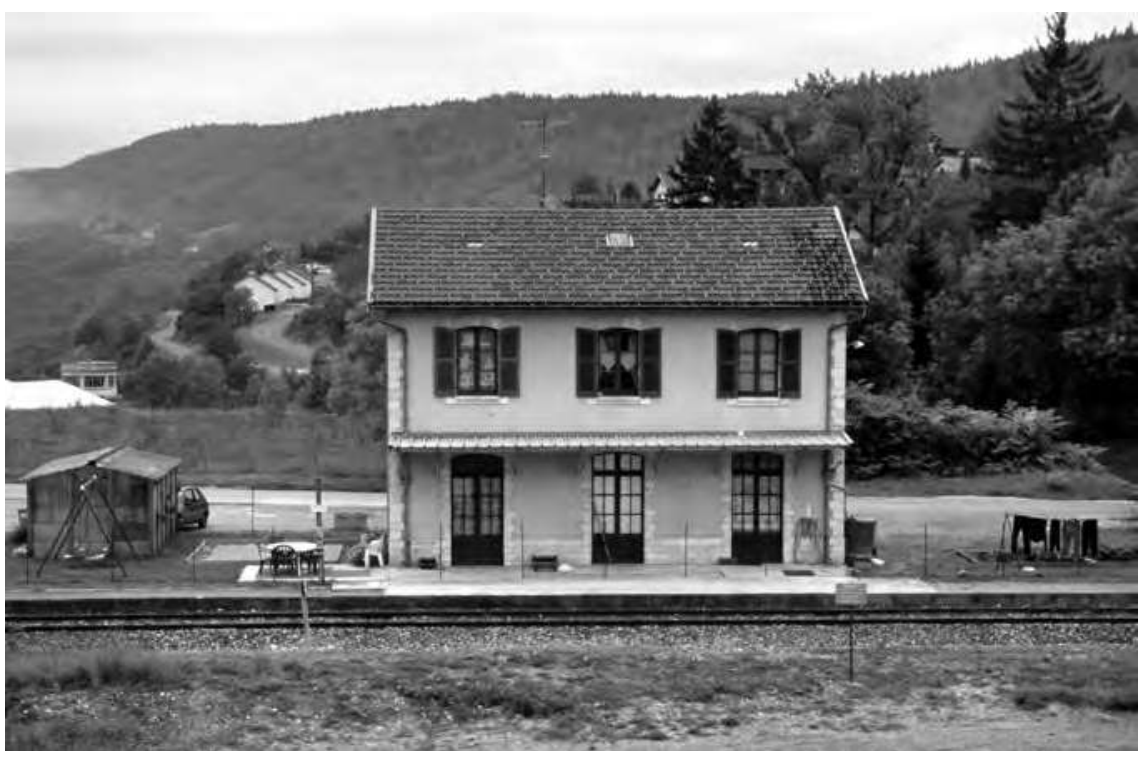

- Figure 5. Gare de $3^{\mathrm{e}}$ classe de Morbier (Jura). Cliché Yves Sancey ${ }^{\odot}$ Région Franche-Comté, Inventaire du patrimoine, ADAGP, 2004. 
Figure 6 • Maison de garde. Dessin imprimé, s.n., [1919]. Publié dans : Compagnie des Chemins de Fer de Paris à Lyon et à la Méditerranée, Ligne de Morez à Saint-Claude.

Rapport sur l'exécution des travaux et les dépenses effectuées, Bourges, Impr. Vve Tardy-Pigelet et Fils, 1919, pl. XLVII. Cliché Jérôme Mongreville ๑ Région Franche-Comté, Inventaire du patrimoine, ADAGP, 2000
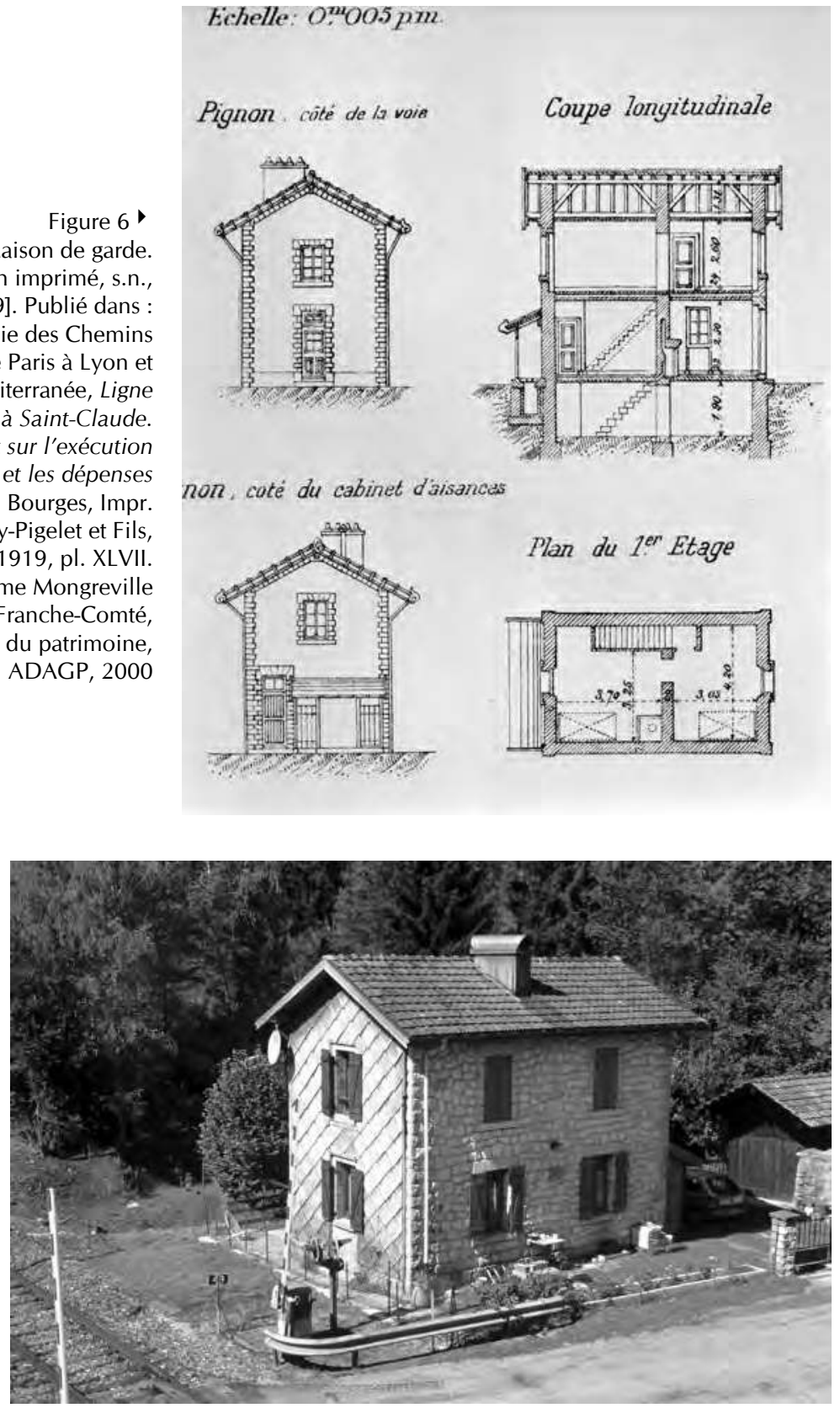

- Figure 7. Maison de garde-barrière et passage à niveau $n^{\circ} 49$, La Rixouse (Jura). Cliché Yves Sancey ${ }^{\odot}$ Région Franche-Comté, Inventaire du patrimoine, ADAGP, 2004 


\section{La question des auteurs}

Cette problématique des modèles et des plans type pose la question des auteurs. Lorsqu'une maison de garde-barrière est construite en X exemplaires suivant un modèle préétabli, quel en est l'auteur ? De même pour un bâtiment de voyageurs, un pont, etc. L'absence de documentation sur les ouvrages, pris individuellement, complique encore cette recherche de paternité.

Procédons par étapes et déterminons tout d'abord les acteurs de la construction pour une ligne d'intérêt général telle celle d'Andelot - La Cluse. En fait, et nonobstant le cadre fourni par la loi de 1842, plusieurs acteurs se distinguent suivant les époques et les cas de figure.

Premier cas : le projet est établi et réalisé par l'administration des Ponts et Chaussées.

Le premier tronçon Oyonnax - La Cluse de la section Saint-Claude - La Cluse relève de ce cas de figure : l'avant-projet en a été dressé par les ingénieurs des Ponts et Chaussées Toussaint (pour la partie jurassienne) et Monnet (pour celle dans l'Ain), le tout sous le contrôle de l'ingénieur en chef Picquenot, qui en dirige également la réalisation, une loi de 1880 ayant autorisé l'État à entreprendre à ses frais la construction (infrastructure et superstructure) de la section Saint-Claude - La Cluse.

Deuxième cas : le projet est établi par les Ponts et Chaussées et réalisé, partiellement ou totalement, par le concessionnaire.

Il en est ainsi du tronçon Saint-Claude - Oyonnax construit dans le prolongement du précédent. La section Saint-Claude - La Cluse ayant été rétrocédée au PLM dans le cadre des conventions de 1883, l'État a invité la compagnie à entreprendre les travaux de superstructure ${ }^{14}$ moyennant remboursement des frais engagés, déduction faite d'une retenue forfaitaire de 25000 francs par kilomètre. On retrouve ici le cas de figure imposé par la loi de 1842 (travaux d'infrastructure assurée par l'État, de superstructure par les compagnies) mais vite abandonné.

Troisième cas : le concessionnaire réalise projet et construction.

Cela semble être le cas du tronçon Morez - Saint-Claude, où un projet initial des Ponts et Chaussées est repris et modifié par l'ingénieur en chef du PLM, Paul Séjourné, qui en orchestre la construction. Il en fut de même, apparemment, à l'origine de la ligne, pour l'embranchement de Champagnole attribué à l'ingénieur du PLM Lucien-Hippolyte Vertray, sous la direction de

14- L'art. 6 des conventions des 26 mai et 9 juillet 1883 spécifie que la compagnie « exécutera ou achèvera, dans l'ordre qui lui sera indiqué par l'Administration supérieure, les travaux de toutes [les lignes concédées] ». 
son ingénieur en chef, Adrien Ruelle, et sous le contrôle de celui des Ponts et Chaussées Laborie. On s'interroge à ce propos sur le rôle exact des ingénieurs du contrôle.

Quel que soit le cas de figure, la question se pose ensuite du partage des responsabilités entre ingénieur ordinaire et ingénieur en chef.

Ainsi, le grand viaduc de Morez peut être attribué à Paul Séjourné, qui l'évoque dans sa publication en six volumes sur les Grandes vôutes, parue de 1913 à $1916^{15}$. Mais qu'en est-il des huit autres viaducs de cette section ? Et des tunnels et autres ouvrages d'art ? Sachant, par ailleurs, que quatre ingénieurs ordinaires se sont succédé auprès de Séjourné de 1903 à 1912 !

Finalement, l'auteur le moins contestable semble être l'exécutant, c'està-dire l'entrepreneur, connu par le procès-verbal d'adjudication et par ceux de réception (sans parler des réclamations en tous genres conservées dans les dossiers). Mais, là encore, rien n'est assuré : le rapport rédigé sur un accident mortel survenu au tunnel du Champ de Bienne en juin 1909 précise que cet ouvrage relève du quatrième lot de terrassement dit de Valfin, adjugé l'année précédente aux entrepreneurs Mercier et Lafont, mais que ces derniers en ont sous-traité la réalisation " aux nommés Martinoli Paul et Delphilipi Secondo».

Cette question de la paternité de la voie ferrée et de ses constructions demeure donc en suspens. Peut-être le prochain terrain d'étude ferroviaire de l'Inventaire de Franche-Comté, qui - toutes proportions gardées - offrira un élément de comparaison, lui apportera-t-il un début de réponse ? Ce terrain est celui de la ligne à grande vitesse Rhin - Rhône, axe européen majeur pour les liaisons nord-sud et actuellement le plus grand chantier de génie civil français.

15- Paul Séjourné, Grandes voûtes, Bourges, impr. Tardy-Pigelet, 1913-1916, 6 vol., 1732 p., ill. , $32 \mathrm{~cm}$. 


\section{Annexe. Le schéma d'organisation documentaire de l'inventaire}

Une fois la ligne étudiée, la documentation est formalisée et intégrée dans le système documentaire de l'Inventaire et les notices informatiques vont alimenter les bases Mérimée (architecture), Palissy (objets mobiliers) et Mémoire (iconographie). Si l'on ne prend en compte que les éléments architecturaux, l'organisation finale des 127 dossiers est présentée dans l'organigramme joint.

\section{L'arborescence}

À côté du dossier de présentation de l'étude elle-même (cahier des charges, conditions de réalisation, etc.) ont été réalisés trois dossiers collectifs, chacun traitant de la typologie d'une famille d'œuvres :

- sur les gares, haltes de voyageurs et maisons de garde-barrière ;

- sur les viaducs, ponts, passerelles et aqueducs ;

- sur les tunnels.

Les 123 dossiers suivants sont répartis en quatre niveaux :

- un dossier de présentation générale de la ligne ;

- quatre dossiers traitant chacun d'une section de cette voie, le découpage étant donné par son histoire ;

- 118 dossiers d'œuvres dont trois consacrés aux plaques tournantes subsistantes (chacun étant le sous-dossier d'une gare).

Cette arborescence s'emboîtera elle-même peut-être un jour dans un système documentaire plus ample, correspondant à l'étude de l'ensemble du réseau ferroviaire franc-comtois.

Par ailleurs, la définition de l'aire d'étude - la voie ferrée - a nécessité de se poser la question de ses bornes : reliant deux lignes préexistantes, elle "s'accroche " à elles par les gares d'Andelot-en-Montagne au Nord et de Montréal-La Cluse au Sud, chacune se rattachant historiquement et typologiquement à sa ligne d'origine. Toutefois, la ligne Andelot - La Cluse ne peut se concevoir sans sa gare de départ ni sans celle d'arrivée : l'une et l'autre ont donc été intégrées dans l'étude mais lorsque les lignes Dole - Neuchâtel et Bourg-en-Bresse - Genève auront été étudiées, les notices informatiques et les dossiers de ces gares devront être modifiés pour signaler leur double appartenance. 

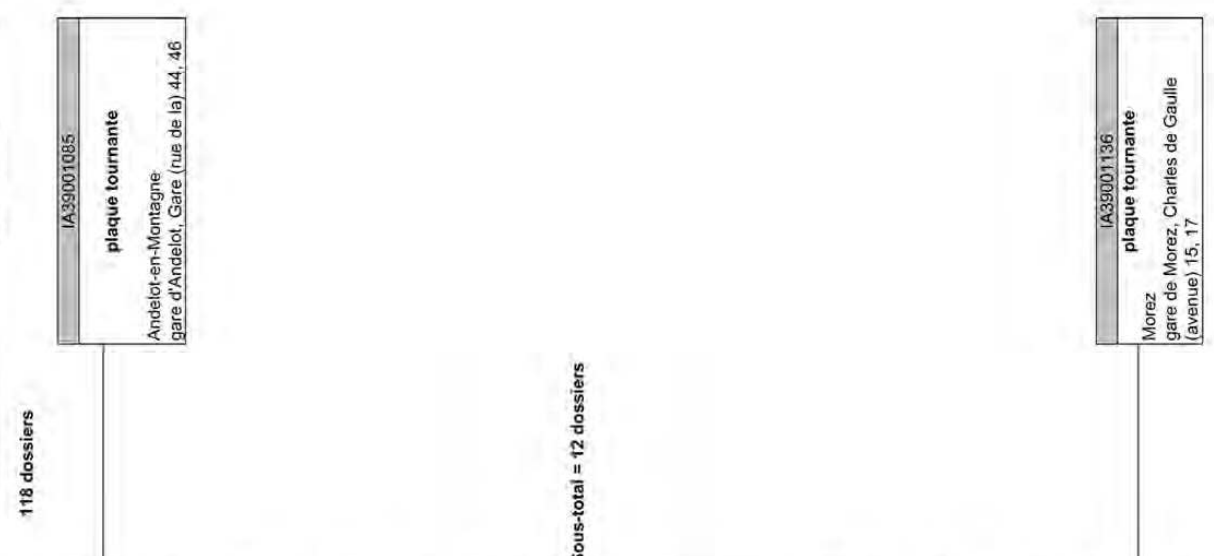

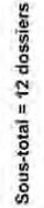

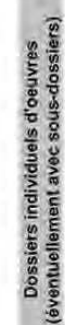

.
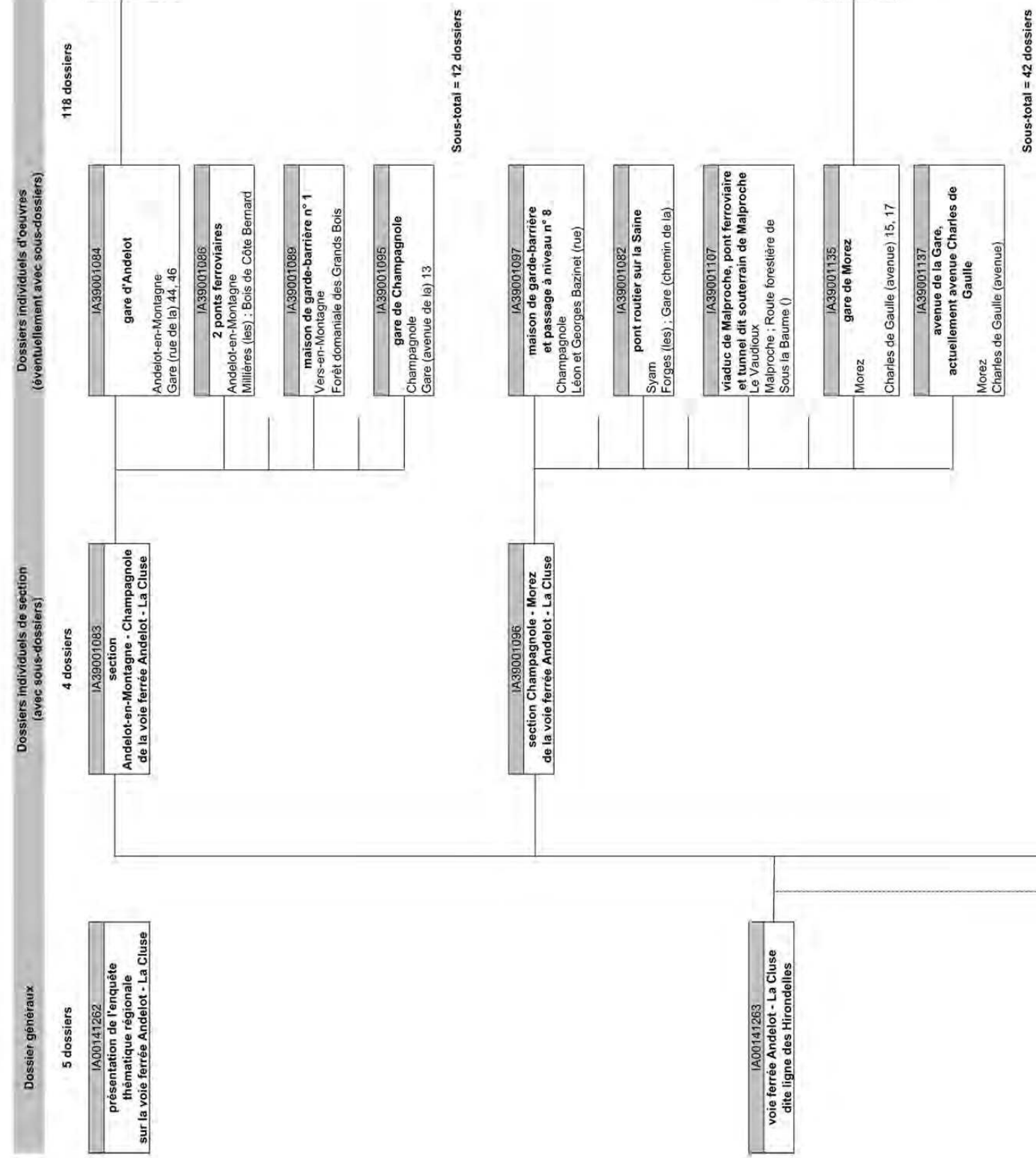

72 - RHCF 40 


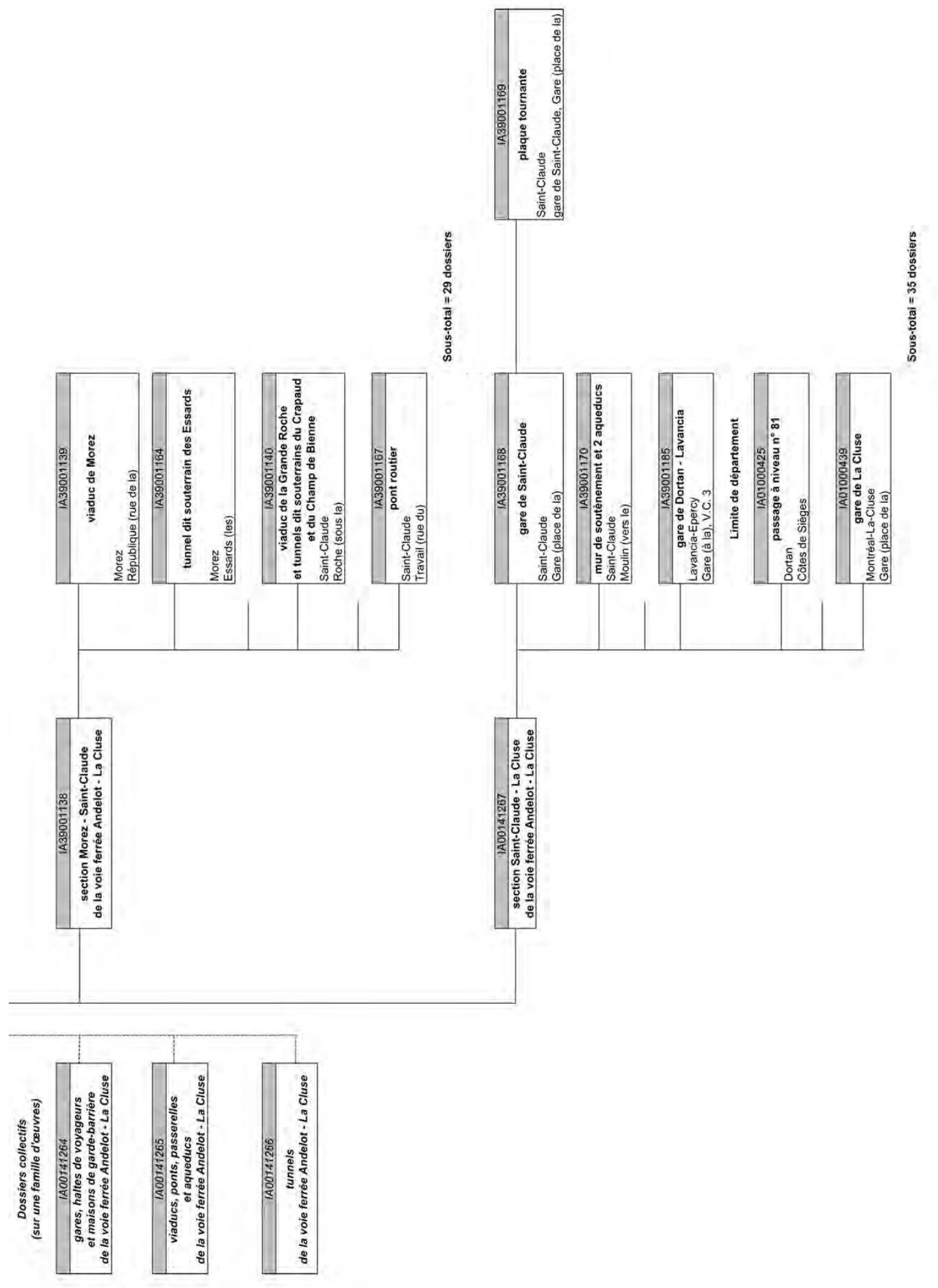


Si l'on évoque la question du traitement des objets mobiliers - dans une acception très large de ce terme -, le schéma organisationnel sera le suivant :

- un dossier de présentation générale des objets de la ligne (donnant notamment ses conditions d'exploitation et de maintenance) ;

- pour certaines gares, un dossier de présentation des objets de la gare ;

- X dossiers d'œuvres consacrés aux objets de ces gares (appareil de voie, serrure centrale, etc.) ;

- X dossiers d'œuvres consacrés à des objets indépendants des gares (téléphone de voie, etc.).

\section{Les incidences du découpage géographique}

Pour un réseau, les limites imposées par un découpage géographicoadministratif sont vite dépassées. Ainsi, la voie ferrée Andelot - La Cluse concerne deux départements (Jura et Ain), mais aussi deux régions (FrancheComté et Rhône-Alpes), d'où l'obligation de passer outre le cadre strictement régional sur lequel est basé le travail de l'Inventaire et de travailler en interrégion.

Par ailleurs, chaque document (notice informatique) alimentant les bases d'œuvres Mérimée et Palissy du ministère de la Culture, ainsi que la base iconographique Mémoire (notice informatique et image numérique), est repéré par un numéro d'immatriculation incluant celui du département où se situe l'œuvre. Cette caractéristique implique donc :

- d'obtenir de la seconde région (Rhône-Alpes) un intervalle de numéros d'immatriculation concernant le département de l'Ain pour les dossiers des œuvres qui y sont étudiées et pour les images produites là ;

- d'obtenir de la direction de l'Architecture et du Patrimoine du ministère de la Culture un intervalle de numéros d'immatriculation pour les dossiers à gérer à un niveau supérieur, c'est-à-dire concernant simultanément les deux régions. 